\title{
FAUNA OF PROTECTED AREAS - 3 SPIDERS OF PURNA WILDLIFE SANCTUARY, DANGS, GUJARAT
}

\author{
Manju Siliwal ${ }^{1}$, B. Suresh ${ }^{2}$ and Bonny Pilo ${ }^{3}$ \\ ${ }^{1}$ Research Associate, Wildife Information \& Liaison Development Society, 29/1 Bharathi Colony, Peelamedu, Coimbatore, \\ Tamil Nadu 641004, India. \\ ${ }^{2,3}$ Department of Zoology, Faculty of Science, The Maharaja Sayajirao University, Baroda, Gujarat 390002, India. \\ Email: ${ }^{1}$ manjusiliwal@rediffmail.com, ${ }^{2}$ suved9@hotmail.com, ${ }^{3}$ bonnypilo@satyam.net.in
}

\begin{abstract}
The spider diversity of Purna Wildlife Sanctuary, Dangs, Gujarat as recorded in 2000-2001 is 116 species belonging to 66 genera and 25 families. Family Araneidae was found to be dominating. Rare spiders like Rhene khandalaensis and Stegodyphus mirandus are first records for Gujarat. A burrow of ground Therasphosid was also sighted.
\end{abstract}

\section{Keywords}

Checklist, Dangs, Purna Wildlife Sanctuary, spiders

\section{Introduction}

The northern extremity of Western Ghats in the Dangs District, Gujarat harbours diverse flora and fauna in its tropical moist deciduous forests. Two protected areas, Purna Wildlife Sanctuary and Vansda National Park render protection to the rich biodiversity of this forest. However, the knowledge on the invertebrate fauna in this region is limited. In an isolated attempt Singh and co-workers studied the biodiversity of Vansda National Park in Dangs, Gujarat (Singh et al., 2000). The present study was made to provide a checklist of spiders at Purna Wildlife Sanctuary.

\section{Study area}

Purna Wildlife Sanctuary (205 $\left.51^{\prime}-21^{\circ} 21^{\prime} \mathrm{N} \& 73^{\circ} 32^{\prime}-73^{\circ} 48^{\prime} \mathrm{E}\right)$ (Fig. 1) has a total area of $160.84 \mathrm{~km}^{2}$ and consists of southern Indian tropical moist deciduous forests (Group 3A/C1), which can be further categorized into southern moist deciduous forests and southern dry deciduous forests (Champion \& Seth, 1968; Singh etal., 2000).

\section{Methodology}

The study was carried out in four different ranges; Mahal,
Bheshkari, Bardipada and Kalibel of Purna WLS from October 2000-September 2001. All types of habitat, such as, bamboo forest, teak plantation, riparian zone and grassland were surveyed. Five sampling sites (each of $10 \mathrm{~m}^{2}$ area) were selected at random in each habitat on every visit (2-3 visits in monsoon and post-monsoon season). On an average two hours were spent in each sampling site. Spiders were searched thoroughly in all probable microhabitats e.g. under stones, dead leaves, bushes, branches of trees, near water bodies, trees and tree trunks up to $2 \mathrm{~m}$ above ground level at every sampling site. Unnecessary collection was avoided by noting down the common spider species in the field. Spiders, which were difficult to identify in the field were collected and preserved in $70 \%$ ethyl alcohol and appropriately labeled with sample number, date, locality and remarks.

To avoid contamination, alcohol in all the vials was changed once in the laboratory. Spiders were identified after detailed microanalysis under WILD ${ }^{\mathrm{TM}}$ Stereomicroscope with the help of standard monographs and literature (Pocock, 1900; Kaston, 1978; Tikader, 1977, 1980, 1982, 1987; Tikader \& Biswas, 1981). Only sexually mature spiders were identified to the species level, while spiderlings/immature spiders were identified only up to the genus level. Identified spiders were labeled and kept individually in small polypropylene vial $(25 \times 50 \mathrm{~mm})$ containing $70 \%$ ethyl alcohol. Genus and species name and their placement in the repective family is according to Platnick (2002).

All mature specimens are deposited at the Departmental Museum, Department of Zoology, Faculty of Science, M.S. University of Baroda, Gujarat, and is in the charge of the second author.

\section{Observations}

Purna Wildlife Sanctuary provides diverse habitat to various spider species. The study revealed the occurrence of 116 species 
belonging to 66 genera and 25 families of spider (Table 1), of which $47.41 \%$ were weavers $(6.89 \%$ ground weavers \& $40.52 \%$ foliage weavers) while $52.59 \%$ were non-weavers (31.9\% foliage hunters \& $20.69 \%$ ground hunters). Among the 116 species of spiders, eight are probably new species (Cyclosa sp. Gasteracantha sp., Larinia sp., Lyssomanes sp., Tinus sp., Scytodes sp., Poltys sp. and Steatoda sp.), which will be published separately after comparative taxonomic work. Rare spiders like Rhene khandalaensis and Stegodyphus mirandus are first records for Gujarat.

Amongst these 25 families, the most dominant family was Araneidae (12 genera \& 28 species), ten families were represented by single species (Table 1). Occurrence of high number of Araneids could be due to mixed vegetation of the forest, which provides enough space to build webs of different sizes and protection from their predators. During the survey, one burrow of a ground theraphosid and a few spiders belonging to family Amaurobiidae and Zodariidae, which are supposedly uncommon in India were also observed. This, shows that the forest ecosystem supports habitat for uncommon and rare spiders.

The results show that the spider diversity in this forest is much higher and more intensive studies may yield more information about the diverse Araneae fauna of this area.

\section{Acknowledgement}

Financial assistance from Gujarat Ecology Commission for the project "Conservation of the Rare and Endangered Biodiversity of Gujarat" under which this study was carried out is gratefully acknowledged. We are thankful to Mr. Anil Johari (Conservator of Forests, Surat Circle), Mr. Mukesh Kumar (Dy. Conservator of Forests, North Dangs) for granting permission to conduct the survey in Purna Wildlife Sanctuary.

\section{References}

Champion, H.G. and S.K. Seth. (1968). A Revised Survey of the Forest Types of India. Government of India Press, New Delhi, 404pp. Kaston, B. J. (1978). How to know the spiders. $3^{\text {rd }}$ ed. Wm.C. Brown Co., Dubuque, Iowa, 289pp.

Platnick, N.I. (2002). The World Spider Catalog, version 3.0. American Museum of Natural History, online at http:// research.amnh.org/entomology/spiders/catalog81-87/index.html (downloaded September/October, 2002)

Pocock, R.I. (1900). The Fauna of British India, Arachnida. Taylor and Francis, London, 279pp.

Singh, H.S., B.R. Raval, B.H. Patel, K. Tatu, D. Patel, R. Vyas and B.H. Patel (2000). Biodiversity Study on Vansda National Park (A comprehensive ecological and socio-economic study). GEER Foundation, Gandhinagar, 176pp.

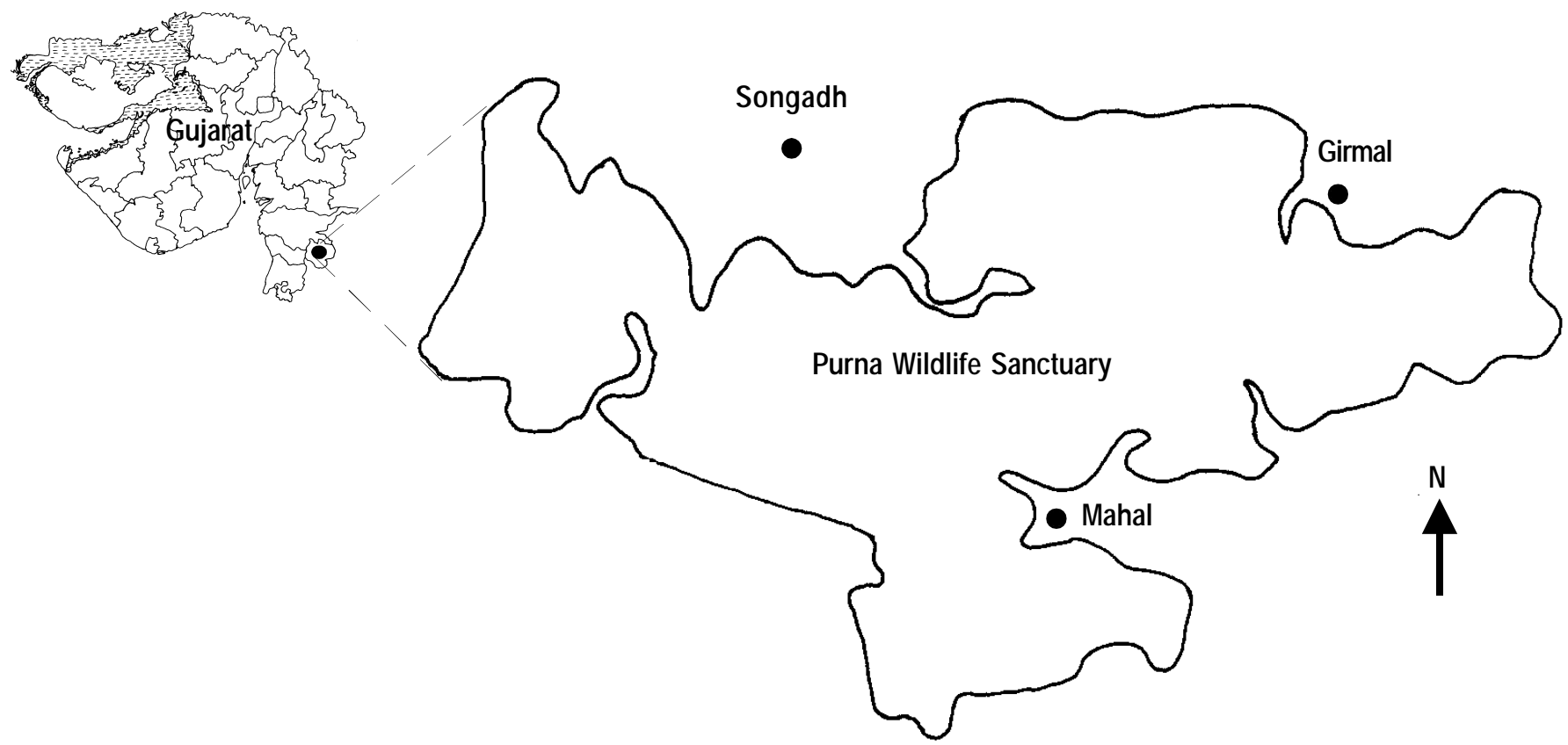

Figure 1. Map of Purna Wildlife Sanctuary 
Table 1. Systematic list of Spiders from Purna Wildlife Sanctuary, Dangs, Gujarat

\begin{tabular}{|c|c|c|c|}
\hline Scientific name & Habit & No. of spiders observed* & No. of spiders collected (tag no.) \\
\hline \multicolumn{4}{|l|}{ Agelenidae C.L. Koch, 1837} \\
\hline Agelena sp. & Ground Weaver & - & 1F (Spi-308-1) \\
\hline \multicolumn{4}{|l|}{ Amauribiidae Thorell, 1870} \\
\hline Amaurobius sp. & Ground Weaver & - & $1 F($ Spi-303-01), $1 S$ \\
\hline \multicolumn{4}{|l|}{ Araneidae Simon, 1895} \\
\hline$\overline{\text { Araneus bilunifer Pcocok }}$ & Foliage Weaver & $3 \mathrm{~F}, 1 \mathrm{M}$ & $2 F($ Spi-92-02, 03) \\
\hline Araneus pahalgaonensis Tikader \& Bal & Foliage Weaver & - & 1F (Spi-312-01) \\
\hline Argiope aemula (Walckenaer) & Foliage Weaver & $9 F$ & - \\
\hline Argiope anasuja Thorell & Foliage Weaver & $12 \mathrm{~F}$ & - \\
\hline Argiope pulchella Thorell & Foliage Weaver & $2 \mathrm{~F}$ & - \\
\hline Cyclosa confraga (Thorell) & Foliage Weaver & $1 \mathrm{~F}$ & $1 F($ Spi-97-01) \\
\hline Cyclosa mulmeinensis (Thorell) & Foliage Weaver & - & $1 F($ Spi-301-01) \\
\hline Cyclosa sp. & Foliage Weaver & - & $1 \mathrm{M}($ Spi-280-02) \\
\hline Cytrophora cicatrosa (Stoliczka) & Foliage Weaver & $15 F, 3 M$ & - \\
\hline Cytrophora citricola (Forskal) & Foliage Weaver & $3 \mathrm{~F}, 2 \mathrm{M}$ & - \\
\hline Eriovixia excelsa (Simon) & Foliage Weaver & $2 \mathrm{~F}$ & $2 F($ Spi-30-14, 15) \\
\hline Eriovixia laglaizei (Simon) & Foliage Weaver & $6 \mathrm{~F}$ & 2F (Spi-34-12, 13) \\
\hline Gasteracantha hasseltii C.L. Koch & Foliage Weaver & $25 \mathrm{~F}$ & - \\
\hline Gasteracantha kuhlii C.L. Koch & Foliage Weaver & $2 F$ & $1 F($ Spi-300-01) \\
\hline Gasteracantha unguifera Simon & Foliage Weaver & $3 F$ & - \\
\hline Gasteracantha sp. & Foliage Weaver & - & $1 F($ Spi-299-01) \\
\hline Larinia phthisica (L. Koch) & Foliage Weaver & - & $1 F($ Spi-39-02) \\
\hline Larinia sp. & Foliage Weaver & - & 1F (Spi-308-01), 1S \\
\hline Neoscona bengalensis Tikader \& Bal & Foliage Weaver & - & 3F (Spi-75-01, 02, 03) \\
\hline Neoscona molemensis Tikader \& Bal & Foliage Weaver & - & $1 \mathrm{M}(\mathrm{Spi}-313-01)$ \\
\hline Neoscona mukerjei Tikader & Foliage Weaver & $12 \mathrm{~F}, 7 \mathrm{M}$ & - \\
\hline Neoscona nautica (C.L. Koch) & Foliage Weaver & - & $1 F($ Spi-296-03) \\
\hline Neoscona sinhagadensis (Tikader) & Foliage Weaver & - & $1 \mathrm{~F}(\mathrm{Spi}-22-01)$ \\
\hline Neoscona theisi (Walckenaer) & Foliage Weaver & $15 \mathrm{~F}, 5 \mathrm{M}$ & - \\
\hline Parawixia dehaanii (Doleschall) & Foliage Weaver & $3 F$ & $1 F($ Spi-25-04) \\
\hline Poltys sp. & Foliage Weaver & - & $1 F($ Spi-293-01) \\
\hline Zygeilla indica Tikader \& Bal & Foliage Weaver & $2 \mathrm{~F}, 1 \mathrm{M}$ & $1 F($ Spi-32-06) \\
\hline Zygeilla melanocrania (Thorell) & Foliage Weaver & - & $1 F($ Spi-31-04) \\
\hline \multicolumn{4}{|l|}{ Clubionidae Wagner, 1887} \\
\hline Clubiona filicata Cambridge & Foliage Hunter & $3 \mathrm{~F}, 1 \mathrm{M}$ & - \\
\hline \multicolumn{4}{|l|}{ Eresidae C.L. Koch, 1851} \\
\hline Stegodyphus tibialis (O.P. Cambridge) & Foliage Weaver & - & 1F (Spi-314-01) \\
\hline Stegodyphus sarasinorum Karsch & Foliage Weaver & $25 F, 4 M$ & 3F (Spi-316-03,04,07), $1 \mathrm{M}$ (Spi-316-05) \\
\hline Stegodyphus mirandus Pocock & Foliage Weaver & - & 1F (Spi-315-01), 1M (Spi-315-02) \\
\hline \multicolumn{4}{|l|}{ Filistatidae Ausserer, 1867} \\
\hline Filistata sp. & Ground Weaver & - & 1S (Spi-302-01) \\
\hline \multicolumn{4}{|l|}{ Gnaphosidae Pocock 1898} \\
\hline Callilepis sp. & Ground Hunter & - & 1F (Spi-unid-15) \\
\hline Drassodes sp. & Ground Hunter & - & 1F(Spi-unid-21) \\
\hline Gnaphosa poonaensis Tikader & Ground Hunter & - & $2 F($ Spi-169-03, 04) \\
\hline Gnaphosa stoliczka O. P. Cambridge & Ground Hunter & - & $1 F($ Spi-317-01) \\
\hline Scopoides sp. & Ground Hunter & - & 1F (Spi-unid-22) \\
\hline Sosticus sundargarhensis Gajbe & Ground Hunter & - & $2 F($ Spi-283-09, 10) \\
\hline \multicolumn{4}{|l|}{ Hersiliidae Thorell, 1870} \\
\hline Hersilia savignyi Lucas & Foliage Hunter & $5 F, 2 M$ & 2F (Spi-91, 05), 1M (Spi-90-03) \\
\hline \multicolumn{4}{|l|}{ Linyphiidae Blackwall, 1859} \\
\hline Linyphia sikkimensis Tikader & Foliage Weaver & - & 2F (Spi-318-01, 02) \\
\hline
\end{tabular}




\begin{tabular}{|c|c|c|c|}
\hline Scientific name & Habit & No. of spiders examined & No. of spiders collected (tag no.) \\
\hline \multicolumn{4}{|l|}{ Lycosidae Sundevall, 1833} \\
\hline Evippa sp. & Ground Hunter & - & 1F (Spi-unid-19) \\
\hline Hippasa lycosina Pocock & Ground Weaver & - & $1 F($ Spi-98-03) \\
\hline Hippasa pisaurina Pocock & Ground Weaver & $11 F$ & $1 F($ Spi-43-05) \\
\hline Hippasa sp. & Ground Weaver & - & $1 F($ Spi-unid-20) \\
\hline Lycosa tista Tikader & Ground Hunter & - & $1 F($ Spi-44-09) \\
\hline Lycosa poonanensis Tikader \& Malhotra & Ground Hunter & - & $1 F($ Spi-221-07) \\
\hline Lycosa madani Pocock & Ground Hunter & - & $2 F($ Spi-89-04, 05) \\
\hline Lycosa pictula Pocock & Ground Hunter & - & $1 F($ Spi-198-03) \\
\hline Lycosa mahabaleshwarensis Tikader \& Malhotra & Ground Hunter & $5 F$ & 3F (Spi-47-09, 10, 11) \\
\hline Pardosa birmanica Simon & Ground Hunter & $16 \mathrm{~F}, 29 \mathrm{M}$ & - \\
\hline Pardosa sumatrana (Thorell) & Ground Hunter & - & 2F (Spi-201-02, 03) \\
\hline Pardosa pusiola (Thorell) & Ground Hunter & - & $1 F($ Spi-114-01) \\
\hline Pardosa sp. & Ground Hunter & - & $1 \mathrm{M}$ (Spi-unid-18) \\
\hline \multicolumn{4}{|l|}{ Miturgidae Simon, 1885} \\
\hline Cheiracanthium sp. & Foliage Hunter & - & 1M (Spi-unid-14) \\
\hline \multicolumn{4}{|l|}{ Oecobiidae Blackwall, 1862} \\
\hline Oecobius putus Cambridge & Foliage Weaver & $12 F, 5 M$ & $\begin{array}{l}2 F(\text { Spi-128-03, 04), } \\
3 \mathrm{M} \text { (Spi-128-02, 05) }\end{array}$ \\
\hline \multicolumn{4}{|l|}{ Oxyopidae Thorell, 1870} \\
\hline$\overline{\text { Oxyopes chittrae Tikader }}$ & Foliage Hunter & - & 1F (Spi-319-01) \\
\hline Oxyopes ratnae Tikader & Foliage Hunter & - & $1 F($ Spi-182-02) \\
\hline Oxyopes shweta Tikader & Foliage Hunter & $5 F$ & - \\
\hline Oxyopes sp. & Foliage Hunter & - & 1F(Spi-unid-16) \\
\hline Peucetiasp. & Foliage Hunter & - & $1 F($ Spi-unid-17) \\
\hline \multicolumn{4}{|l|}{ Philodromidae Thorell, 1870} \\
\hline$\overline{\text { Philodromus bhagirathi Tikader }}$ & Foliage Hunter & - & 1F (Spi-320-01) \\
\hline Thantanus sp. & Foliage Hunter & - & 1F(Spi-unid-13) \\
\hline Tibellus elongatus Tikader & Foliage Hunter & $3 F, 1 M$ & $\begin{array}{l}\text { 3F (Spi-263-15, 16, 17), } \\
1 \mathrm{M}(\text { Spi-263-14) }\end{array}$ \\
\hline Tibellus pashanensis Tikader & Foliage Hunter & - & $1 F(S p i-275-04)$ \\
\hline Tibellus pateli Tikader & Foliage Hunter & - & $1 F($ Spi-270-07) \\
\hline Thantaus dhakuricus Tikader & Foliage Hunter & $3 F$ & $2 \mathrm{~F}($ Spi-242- 05, 06) \\
\hline Tibellus sp. & Foliage Hunter & - & $\begin{array}{l}\text { 3F (Spi-unid-12-01, 02, 03), } \\
\text { 2M (Spi-unid-12-04, 05) }\end{array}$ \\
\hline \multicolumn{4}{|l|}{ Pholcidae C.L. Koch, 1851} \\
\hline Artema atlanta Walckenaer & Foliage Weaver & $3 F$ & - \\
\hline Crossopriza lyoni (Blackwall) & Foliage Weaver & $9 F$ & - \\
\hline Pholcus sp. & Foliage Weaver & $3 \mathrm{~F}$ & 1F (Spi-unid-11) \\
\hline \multicolumn{4}{|l|}{ Pisauridae Simon, 1890} \\
\hline Pisaura gitae Tikader & Foliage Hunter & $3 \mathrm{~F}$ & 1F (Spi-99-08) \\
\hline Tinus sp. 1 & Foliage Hunter & - & $1 F($ Spi-unid-04) \\
\hline Tinus sp. 2 & Foliage Hunter & - & $1 F($ Spi-323-01) \\
\hline \multicolumn{4}{|l|}{ Salticidae Blackwall, 1841} \\
\hline Asemonea tenuipes (O.P. Cambridge) & Foliage Hunter & - & $1 F($ Spi-321-01) \\
\hline Bianor sp. & Ground Hunter & - & 2F (Spi-unid-10-01,02) \\
\hline Hyllus semicupreus (Simon) & Foliage Hunter & - & $1 F($ Spi-218-05) \\
\hline Lyssomanes sp. & Foliage Hunter & - & $1 F($ Spi-310-01) \\
\hline Marpissa sp. & Ground Hunter & - & 1F (Spi-unid-09) \\
\hline Myrmarachne bengalensis Tikader & Ground Hunter & - & 2F (Spi-288-09) \\
\hline Plexippus paykullii (Audouin) & Ground Hunter & $2 \mathrm{~F}, 3 \mathrm{M}$ & - \\
\hline Rhene khandalaensis Tikader & Ground Hunter & - & 2F (Spi-309-01) \\
\hline Phintella vittata (C.L. Koch) & Ground Hunter & - & $1 F($ Spi-230-06) \\
\hline Phintella sp. & Ground Hunter & - & 1F (Spi-unid-08) \\
\hline
\end{tabular}




\begin{tabular}{|c|c|c|c|}
\hline Scientific name & Habit & No. of spiders examined & No. of spiders collected (tag no.) \\
\hline Telamonia dimidiata (Simon) & Foliage Hunter & - & $1 F($ Spi-95-04) \\
\hline \multicolumn{4}{|l|}{ Scytodidae Blackwall, 1864} \\
\hline$\overline{\text { Scytodes fusca Walckenaer }}$ & Foliage Hunter & - & 1F (Spi-213-06) \\
\hline Scytodes sp. & Foliage Hunter & - & 1F (Spi-175-01) \\
\hline Scytodes thoracica (Latreille) & Foliage Hunter & $3 F$ & $2 F($ Spi-324-01) \\
\hline \multicolumn{4}{|l|}{ Sparassidae Bertkau, 1872} \\
\hline Olios sp. & Foliage Hunter & - & 1M (Spi-unid-06) \\
\hline Heteropoda sp. & Foliage Hunter & - & 1M (Spi-unid-07) \\
\hline \multicolumn{4}{|l|}{ Tetragnathidae Menge, 1866} \\
\hline Leucauge decorata (Blackwall) & Foliage Weaver & $2 \mathrm{~F}$ & 1F (Spi-14-03) \\
\hline Leucauge tessellata(Thorell) & Foliage Weaver & $3 \mathrm{~F}, 2 \mathrm{M}$ & - \\
\hline Nephila kuhlii Doleschall & Foliage Weaver & $2 F$ & 1F (Spi-322-01) \\
\hline Nephila pilipes (Fabricius) & Foliage Weaver & $27 \mathrm{~F}, 22 \mathrm{M}$ & - \\
\hline Tetragnatha sp. & Foliage Weaver & - & 1F (Spi-unid-03) \\
\hline \multicolumn{4}{|l|}{ Theraphosidae Thorell, 1870} \\
\hline Unidentified species \# & Ground Weaver & - & - \\
\hline \multicolumn{4}{|l|}{ Theridiidae Sundevall, 1833} \\
\hline Argyrodes ambalikae Tikader & Foliage Weaver & - & $1 F($ Spi-53-05) \\
\hline Argyrodes xiphias Thorell & Foliage Weaver & - & 1F (Spi-323-01) \\
\hline Argyrodes gazingensis Tikader & Foliage Weaver & - & $1 F($ Spi-324-01) \\
\hline Steatoda sp. & Ground Weaver & - & $1 F($ Spi-298-01) \\
\hline Theridion sp. & Foliage Weaver & - & 1F (Spi-unid-05) \\
\hline \multicolumn{4}{|l|}{ Thomisidae Sundevall, 1833} \\
\hline Camaricus khandalaensis Tikader & Foliage Hunter & - & 1F (Spi-306-02), 1M (Spi-306-01) \\
\hline Oxytate elongata (Tikader) & Foliage Hunter & - & 1F (Spi-325-03) \\
\hline Monaeses parvati Tikader & Foliage Hunter & $5 F$ & $1 F($ Spi-269-07) \\
\hline Runcinia ghorpadei Tikader & Foliage Hunter & - & $1 F($ Spi-326-01) \\
\hline Thomisus shivajiensis Tikader & Foliage Hunter & - & $1 F($ Spi-16-11) \\
\hline Thomisus cherapunjeus Tikader & Foliage Hunter & $3 F$ & $1 F($ Spi-13, 26) \\
\hline Thomisus elongatus Stoliczka & Foliage Hunter & - & $2 \mathrm{~F}($ Spi-327-02, 03) \\
\hline Thomisus sp. & Foliage Hunter & - & 1F (Spi-unid-02) \\
\hline Thomisus pugilis Stoliczka & Foliage Hunter & - & 1F (Spi-239-04) \\
\hline Xysticus minutus Tikader & Foliage Hunter & $6 \mathrm{~F}$ & - \\
\hline \multicolumn{4}{|l|}{ Uloboridae Thorell, 1869} \\
\hline$\overline{\text { Uloborus danolius Tikader }}$ & Foliage Weaver & $5 F, 2 M$ & - \\
\hline Uloborus khasiensis Tikader & Foliage Weaver & $6 \mathrm{~F}$ & 3F (Spi-196-03, 04, 05) \\
\hline \multicolumn{4}{|l|}{ Zodariidae Thorell, 1881} \\
\hline Lutica sp. & Ground Hunter & - & 1F(Spi-unid-01) \\
\hline
\end{tabular}

Note: * - Spiders not collected; \# - indirect evidence of its occurrence; F - female; M - male; S - subadult; Spi-unid- unidentified species

Tikader, B.K. (1977). Studies on spider fauna of Andaman and Nicobar Islands, Indian Ocean. Records of Zoological Survey of India 72(1-4): 157-212.

Tikader, B.K. (1980). Fauna of India: Spider (Thomisidae and Lycosidae). Zoological Survey of India, Calcutta, 443pp.

Tikader, B.K. (1982). Fauna of India: Spider (Araneidae and Gnaphosidae). Zoological Survey of India, Calcutta, 533pp.

Tikader, B.K. (1987). Handbook: Indian Spiders. Zoological Survey of India, Calcutta, 251pp.
Tikader, B.K. and B. Biswas. (1981). Spider fauna of Calcutta and vicinity. Part I. Records of Zoological Survey of India, Occasional Paper 30: 1-148. 